\title{
Medical Research in Infants and Children in the Eighties: Analysis of Rejected Protocols
}

\author{
GIDEON KOREN AND ANNE PASTUSZAK \\ Division of Clinical Pharmacology, Department of Pediatrics, and the Human Experimentation Review \\ Committee, the Research Institute, The Hospital for Sick Children, Toronto; Departments of Pediatrics and \\ Pharmacology, University of Toronto, Toronto, Ontario, Canada
}

\begin{abstract}
We reviewed the 351 protocols dealing with research in infants and children in our institute between July 1982 and August 1988 to identify ethical issues that complicate the execution of pediatric research. Of the 16 rejected protocols $(4.5 \%) 12$ were drug studies, three dealt with the nature or course of disease states and one was in the area of behavioral sciences. Drug studies were significantly more likely to be rejected than all other studies. The most common reason for rejection $(n=10)$ were major scientific flaws that, according to the Committee, would result in inability of the study to answer the questions posed by the researchers. In nine cases the Committee judged a study to be physically invasive without a direct benefit to the involved infant/child. In three cases, the Committee rejected a study because patients with serious medical conditions might be randomized to receive placebo and not a drug that, based on current knowledge from adults, would possibly improve their condition. In three protocols, current antimicrobial therapy covered all pathogens causing the infection and the proposed new therapy could not improve the prognosis further but only be equal or inferior. Researchers who had more than one protocol rejected had significantly more protocols submitted (7.17 $\pm 1.35)$ than those who had only one rejection $(1.86 \pm$ $0.36, p<0.0005)$ or than the 10 researchers with the highest number of studies without a single rejection (4.2 \pm $0.4, p<0.05)$. These numbers suggest that scientists who perform more research are more likely to stand at the front line of unresolved ethical issues. (Pediatr Res 27: 432-435, 1990)
\end{abstract}

\section{Abbreviations}

HERC, The Human Experimentation Review Committee $\mathrm{OC}$, oral contraceptive

In 1957, Goodlin and Kaiser reported on the effects of ammonium chloride given to pregnant women before labor on neonatal $\mathrm{pH}$ (1). They administered large doses of the compound to healthy pregnant women who volunteered to participate "after the nature of the study was explained to them." Neonatal $\mathrm{pH}$ was acidic in all cases, and as low as 7 in some babies. Seeking to answer more questions about the time kinetics of transplacental acidification, the same group repeated the study in a slightly different design (2). Reading these two scientific documents is a shocking experience to anyone who participates in medical re-

Received August 29, 1989; accepted December 19, 1989.

Correspondence Gideon Koren, M.D., Division of Clinical Pharmacology, The Hospital for Sick Children, 555 University Ave., Toronto, Ontario, M5G 1X8, Canada. search in infants and children, and it may serve as a reference for the long way medical ethics have evolved in the last two decades.

After the Helsinki Convention in 1964, which aimed at crystallizing rules to prevent human experimentation of the nature performed by the Nazis during World War II (3), each medical institution in countries that signed the accord has to submit all protocols dealing with human research to an institutional review board.

To date, medical research in fetuses, infants, and children in North America is controlled by strict guidelines that aim at protecting the nonconsenting, developing human being. Although the need for such protection is self explanatory, it is clearly a two-edged sword as it may prevent or retard the understanding of physiology and pathology during development and the establishment of needed therapeutic modalities for infants and children, because available research methods may be invasive and unethical in children. The lack of understanding of the physiology and control of pain in the newborn may serve as such an example, recently discussed in medical journals $(4,5)$.

Serving in a human experimentation review board of a large pediatric hospital, we are struggling with the limits of ethical boundaries of medical research in infants and children in an attempt to find solutions that will protect pediatric patients while allowing optimal development of research crucially needed for improvement of pediatric care. In this study, we analyzed protocols rejected by our review board over a 6-y period in an attempt to offer the reader a "window" into the ethical issues inherent to pediatric research in a major pediatric center.

\section{STUDY DESIGN}

The Hospital for Sick Children in Toronto is a 700-bed tertiary care pediatric hospital serving the Greater Toronto area. In addition, it is the base for the Department of Pediatrics of the University of Toronto, and most pediatric teaching is conducted there. The HERC meets monthly throughout the year to review and approve all research projects involving human subjects. The committee is comprised of three to four academic pediatricians (including the chairman), a surgeon, an anesthetist, a clinical pharmacologist (pediatrician), a clinical pharmacist, a nurse, a psychologist, a clergyman/woman, a professor of law, and a representative of the public (generally a parent of a child who experienced long hospitalization).

Every submitted study is reviewed for its scientific merits as well as for its ethicality. Before September 1985, both functions were conducted simultaneously by the HERC; after September 1985 , a protocol has been first peer reviewed scientifically by outside experts familiar with the particular area of research. Only after such a process is it reviewed ethically.

Methods of analysis. All studies reviewed by the HERC between July 1982 and August 1988 were analyzed. As a first step we reviewed the minutes summarizing the nature of the study, 
its ethical problems and the decisions by the committee. Subsequently, the complete protocols of all studies that were rejected or not approved during the first discussion were reviewed in depth.

For the purpose of our analysis, we defined "rejection" as nonpermission to conduct the study, either totally, or as proposed; in a few cases, permission was granted only after major revisions that changed the nature of the study and of the questions that could be answered by it.

\section{RESULTS}

During the 6 years analyzed by us, the HERC reviewed 351 protocols of which $16(4.5 \%)$ were rejected. Of the rejected protocols $12(75 \%)$ were drug studies, three $(18 \%)$ dealt with the nature or course of disease states and one $(6 \%)$ was in the area of behavioral sciences. Drug studies were rejected significantly more than any other type of protocol (Table 1). Studies were classified by the reasons for their rejection and one protocol could have been rejected due to more than one argument (Table 2 ). Each reason is documented by one example (cases $1-8$ ). The most common $(n=10)$ was rejection due to major scientific flaws (case 1). In nine cases, the committee judged a study to be physically invasive with risks being above the minimal without a direct benefit to the involved infant/child; in eight of these, the invasiveness involved extra blood sampling (case 2) and in the ninth case, an extra muscle biopsy. In three cases, the committee rejected a study because patients with serious medical conditions might be randomized to receive placebo and not a drug that, based on current knowledge from adults, would possibly improve their condition (case 3 ). In three protocols, current antimicrobial therapy covered all pathogens causing the infection and the

Table 1. Characteristics of rejected protocols

\begin{tabular}{lccc}
\hline \multicolumn{1}{c}{ Type of study } & Total no. & No. rejected $(\%)$ \\
\hline Drug studies & 161 & $12(7.45)$ & $p<0.05^{*}$ \\
Studies in behavioral sciences & 48 & $1(2.1)$ \\
$\begin{array}{l}\text { Physiology, mechanisms and } \\
\text { course of disease }\end{array}$ & 131 & $3(2.3)$ \\
$\begin{array}{l}\text { Studies of medical devices } \\
\text { Total }\end{array}$ & 11 & $0(0)$ \\
\hline
\end{tabular}

$* p<0.05$ when compared to all other studies.

Table 2. Analysis of reasons for rejection of research protocols dealing with children

\begin{tabular}{l}
\hline \multicolumn{1}{c}{ Reasons for rejection } \\
$\begin{array}{l}\text { A mo. of studies } \\
\text { the intended question(s) }\end{array}$ \\
$\begin{array}{l}\text { A physically invasive study with no direct benefit to } \\
\text { infant/child }\end{array}$ \\
$\begin{array}{l}\text { Placebo to patients who may potentially benefit from } \\
\text { the drug }\end{array}$ \\
$\begin{array}{l}\text { Experimental drug may expose the child to risk } \\
\text { greater than current therapy and probably not to }\end{array}$ \\
lesser risk \\
$\begin{array}{l}\text { Emotionally invasive study with no direct benefit to } \\
\text { patient }\end{array}$ \\
$\begin{array}{l}\text { Randomization will pose on parents an unacceptable } \\
\text { situation in the emergency room }\end{array}$
\end{tabular}

proposed new therapy could not improve the prognosis further but only be equal or inferior (case 4). In one case, the committee believed that a behavioral study, if approved, would expose the child to an unacceptable emotional stress in addition to breaching confidentiality (case 5). In a single case, an argument was made against asking parents permission for randomization of seriously ill children to receive one of two modalities at the emergency room (case 6).

In addition to a consent form, each study must include an information sheet for the parents and children that describes the nature, reasons, and procedures included in the study in simple terms that can be understood by lay persons. Invariably, all studies required rephrasing of at least some sentences of the information data, mainly because the language was believed to be too technical or complicated. In only one case, researchers asked and were allowed to omit the information sheet as it would affect the results of the study (case 7).

Although the legal age for informed consent in Canada is 16 $y$ old, the researchers have to seek the assent of school-age children who do understand the nature of the study. A study protocol cannot be initiated in a case where a child does not agree to participate. In one case researchers were allowed to receive consent from girls younger than $16 \mathrm{y}$ who were already taking oral contraceptives without their parents' knowledge (case 8).

Of the 29 researchers participating in the rejected protocols, six had more than one rejection; however, they had significantly more protocols submitted to the committee (mean \pm SD $7.17 \pm$ 1.35) than those who had only one rejection $(1.86 \pm 0.36, p<$ 0.0005 ), or than the 10 researchers with the highest number of studies without a single rejection $(4.2 \pm 0.4, p<0.05)$.

\section{DISCUSSION}

All investigators conducting human experimentation may find themselves in a conflict between their scientific quest and their societal obligation to protect the well-being of the individual. This issue is complicated and sensitive in the case of infants and children, largely due to two elements characteristic of this group of patients:

Consent. Voluntary consent, which is a prerequisite of the Code of Nuremberg (6), cannot be achieved in the pediatric age group. The World Medical Association's Declaration of Helsinki (3) accepts the consent of the legal guardian and this approach has been adopted by virtually ali medical associations; however, in the case of invasive research, without direct benefit to the involved child, parental consent, even when granted, may not be valid as some ethicists claim that children have no societal obligation to contribute to general welfare (7).

Invasiveness-risk. The National Commission for the Protection of Human Subjects of Biomedical and Behavioral Research, established by the American Congress in 1974, has defined the "minimal risk" that should be maintained during pediatric experimentation as "the probability and magnitude of physical and psychological harm that is normally encountered in the daily lives, or in the routine medical or psychological examination of healthy children" (8). These include immunizations, modest changes in diet, and the obtaining of blood and urine specimens. Under this definition a variety of procedures cannot be conducted, and even repeated blood sampling cannot be interpreted as "normally encountered physical harm." The Commission allowed risks to be "more than minimal" when such research holds out the prospect of direct benefit to the child. Again, such a demand would exclude all areas where the research would be beneficial only for future cases, such as pharmacokinetic studies. The dilemma is whether it is right to "protect children from any research risk that is more than minimal and thereby prevent the possible discovery of means to cure or prevent certain serious disorders ..." (9). Although some ethicists would maintain that there is no positive duty to benefit, whereas there is a duty to 
prevent harm, others believe that this approach is abstract and heartless $(9,10)$. The Commission chose a compromise in which this risk must be "a minor increase over minimal risk."

In dealing with this difficult issue the Commission allowed that children might be entered into research involving more than minimal risk and with no direct benefit when: 1) The risk is "a minor increase over the minimal risk." 2) The experience presented by the intervention is "reasonably commensurate with those inherent in medical, psychological or social situation" of the child. 3) The research is likely to yield generalizable knowledge of "vital importance."

In its recent "Guidelines on Research Involving Human Subjects" the Medical Research Council of Canada does not allow pain or discomfort "beyond carefully defined limits" (11). In both the American and Canadian instances, the Institutional Review Board is expected not only to assure that unequivocal ethical boundaries will be maintained, but also to define in each case if the research falls within the definition of "a minor increase over minimal risk" (in the United States), or to define the "limit of pain or discomfort" (in Canada).

In our analysis, we reviewed studies rejected by our HERC. Being the largest pediatric hospital in North America, covering virtually all areas of medical research in infants and children, gave us a wide angle of ethical considerations and allowed identification of ethical issues and their underlying reasons. This review aimed at identifying what is judged unacceptable from the ethical stand point in our institution, and therefore, what is considered to be acceptable research. The discussed research protocols are representative of pediatric research in general and virtually all these studies are published on completion in peer reviewed medical journals. Similar to such committees in North American pediatric institutions, members of our committee act within the federal guidelines (in our case, the Canadian Medical Research Council).

The most common issues in studies rejected by our HERC were scientific inadequacies which were believed to impair the ability of the researchers to answer the research questions. It is generally agreed that a study cannot be ethically acceptable if its scientific integrity is impaired (7). The complexities of study design, including crystallization of hypothesis, methodology and objectives, analysis of sample size, and statistical approach have to be scrutinized to evaluate its ability to answer the research questions.

The common ethical denominator in most of the studies rejected for nonscientific reasons was the perception of risk as being above the minimal, without a direct benefit to the child. Extra blood sampling is undoubtedly the most difficult hurdle, as a variety of physiopathologic and pharmacologic studies assess changes in concentrations of naturally occurring compounds or xenobiotics in the blood. The inability to conduct pharmacokinetic studies in newborn infants with a large number of samples has led us to reevaluate the design of such investigations $(12,13)$ and to set forth new guidelines; in most cases three samples yield dosing information not less accurately than eight blood samples.

In addition to pain and discomfort, there is evidence that in newborn infants the number of blood transfusions correlates with volume of bilood sampled for laboratory tests (14). In older infants, where the depleted volume is less of a problem, the issue of pain and discomfort associated with blood sampling becomes more dominant. Although concurring sampling for research purposes with routine blood testing is the ideal solution, it obviously does not meet the need of a variety of protocols. Inserting an indwelling catheter for sampling may obviate the need for repeated pricking and thus reduce pain and anxiety without significantly increasing the "minimal risk."

The complexities imposed by introducing placebo have caused rejection of several protocols. Although the principal issues of placebo are not different in infants or children from adult experimentation (10), there is a high level of sensitivity to possibly withholding effective therapy from the sick infant or child. The fundamental assumption in designing randomized drug-placebo studies is that the drug has not yet been proven to be more effective than placebo for the condition in question. In its recommendations, the American Commission clearly stated that studies must be performed first in adults (15); however, if adult studies show clear advantage to the drug over the placebo, is it ethical to repeat such a protocol in children? Case 2 reflects such a dilemma, whereupon our HERC judged as unethical a repetition of a drug-placebo study in children with moderate to severe asthma. Yet, by preventing such a study from being conducted, scores of children may be treated with a modality that may be theoretically ineffective in children, unlike in adults. In this case, two different ethical questions have intertwined: 1) is it ethical to repeat an adult study in children? and 2) should placebo be used in drug trials in children, and if so, under what circumstances?

In several cases, our HERC rejected protocols that aimed at testing new antimicrobial drugs. In all instances, the new therapy could not be assumed to improve the outcome of the disease in question when compared to the present treatment of choice. However, the researchers argued that within a relatively short period, bacterial resistance is likely to emerge, thus compromising the treatment of future pediatric cases. Here, a clear conflict between the risk/benefit of the present subjects of research versus future pediatric cases had to be balanced. In all instances our committee believed that its main obligation is to protect present children from having excessive risk, more than protecting future cases from suboptimal therapy.

Similar to the previous examples involving placebo research or invasive procedures, it is apparent that here too the quality of research conducted in infants and children may be scientifically inferior to similar adult projects due to the unique ethical limitations imposed on the research team.

That drug research is more likely to be rejected than any other pediatric experimentation reflects a potentially serious tendency. In all instances, the drugs in question had been tested in adults before the proposed research in children, thus complying with an important prerequisite of the Commission (15). Yet, despite attempts by our HERC to find solutions that would bridge the distance between ethical and scientific needs, protocols of drug research were more likely to contain an issue that could not be resolved. In all of these cases, the research would have some increased risk/discomfort "above minimal" without direct benefit to the participating infants or children. Here, the philosophical question, whether the nonconsenting children have obligations to society (specifically to other children) despite lack of direct benefit to them $(9,10)$, has been answered negatively by the HERC. The question often asked by some members of our committee during such debates is "would you agree that your own child participate in this research protocol?" It has, however, been argued that most of the committee members are health professionals; because they usually identify strongly with the imperative to do research they may be willing to offer their own child, although it is unlikely that their child will be asked to participate. Our experience, however, suggests that in many instances, when the above question was asked, the committee members believed they would not allow such research done on their own children.

Several innovative, noninvasive approaches may advance drug research in infants and children: The use of saliva to measure unbound drug concentrations has recently been proven accurate in the case of theophylline (16) and may help in studying the bioavailability of new slow-releasing theophylline formulations without the need for repeated blood sampling. Unfortunately, this modality does not achicve favorable results with many other drugs (17). Recently developed breath (18) and urine (19) tests may obviate the need for blood in studying developmental aspects of drug mctabolism. The development of micromethods for drug analysis has dramatically decreased the volume of blood needed for such investigations; this is a crucial aspect in studying 
newborn infants and, especially, the preterm baby, due to their very limited blood volume.

Our analysis reveals that in our institution, researchers who had more rejections of protocols were those who performed more research, and therefore were more likely to stand at the front line of unresolved ethical issues. However, the fact that almost $95 \%$ of protocols were approved must mean that in most cases, ethical issues can be resolved and pediatric research can be advanced. Moreover, after excluding the instances where rejection of protocols was based on scientific flaws, it is evident that only about $2 \%$ of studies cannot be conducted due to unresolved ethical issues.

\section{REFERENCES}

1. Goodlin RC, Kaiser JH 1957 The effect of ammonium chloride-induced maternal acidosis on the human fetus at term: I. pH, Hemoglobin, blood gases. Am J Med Sci 233:662-674

2. Kaiser J, Goodlin RC 1958 The effect of ammonium chloride-induced maternal acidosis on the human fetus at term II. Electrolytes. Am J Med Sci 234:549-554

3. World Medical Association 1970 Declaration of Helsinki 1964. In: Beecher H (ed) Research and the Individual: Human Studies. Little Brown Co, Boston

4. Anand KJS, Hickey PR 1987 Pain and its effect on the human neonate and fetus. N Engl J Med 317:1321-1329

5. Rana SR 1987 Pain-a subject ignored. Pediatrics 79:309

6. Trials of War Criminals Before the Nuremberg Military Tribunals: United States Versus Karl Brandt. 1949 US Government Printing Office, Vol 2, p 181

7. Ramsey $\mathrm{P}$ The enforcement of morals: non-therapeutic research on children. Hastings Center Report 6:21-30

8. Public Law July 12, 1974 US Congress $93: 348$

9. Janssen AR 1978 Research involving children. Pediatrics 62:131-136

10. Levine RJ 1986 Ethics and regulation of clinical research, 2nd ed. Urban \& Schwarzenberg, Baltimore, pp 235-256

11. Medical Research Council of Canada 1987 Guidelines on research involving human subjects. Ottawa, Ontario, pp 29-30

12. Koren G, Litwack J, Biggar D 1988 Using infants in drug research where there is no direct benefit to them: a survey of Canadian health professionals serving in ethical committees. Can Med Assoc J 138:899-902

13. Long D, Koren G, James A 1987 Ethics of drug studies in newborn infants: how many samples are needed for pharmacokinetic analysis? J Pediat 111:918-921

14. Blanchette VS, Zipursky A 1984 Assessment of anemia in newborn infants. Clin Perinatol 11:489-507

15. Federal Register January 13, 1978

16. Aviram M, Tal A, Ben-Zvi Z, Gorodischer R 1987 Monitoring theophylline therapy using citric acid-stimulated saliva in infants and children with asthma. Pediatrics 80:894-897

17. Gibaldi M, Prescott LF (eds) 1983 Handbook of Clinical Pharmacokinetics ADIS Press Ltd, New York

18. Lambert GH, Katake AN, Schaeller D 1983 The $\mathrm{CO}_{2}$ breath tests as monitors of the cytochrome P-450 dependent mixed function monooxygenase system. In: MacLeod SM, Okey AB, Spielberg SP (eds) Developmental Pharmacology. Alan R Liss, New York, pp 119-145

19. Grant DM, Tang BK, Kalow W 1983 Variability in caffeine metabolism. Clin Pharmacol Ther 33:591-602

\section{APPENDIX}

The following cases rejected by the HERC exemplify characteristic ethical issues mentioned above.

Case 1: scientific flaws. The researchers wanted to study the difference in adverse effects between two dose levels of an anticoagulant on 100 children older than 2 y. However, statistically, this sample size is too small to show differences of serious bleeding which is extremely uncommon at the dose levels in question.

Case 2: physically invasive. The researchers wished to compare the oral bioavailability of two preparations of erythromycin in small children treated against pertussis. The study would involve multiple blood sampling. The committee believed that there are no potential benefits to the specific child from the measurement of drug levels. Moreover, erythromycin is not believed to change the course of the illness in the treated child but rather to decrease the chance of spreading the disease. The committee suggested to compare the efficacy of the two formulations in eradicating the bacteria; however, the manufacturer that initiated the study did not agree, as the bioavailability data were needed for approval of the new formulation of erythromycin by the regulatory agency.

Case 3: placebo may harm. The protocol intended to randomize at the emergency room children with moderate to severe asthma, already treated with theophylline and $\beta$-adrenergic agonists, to receive steroids or placebo i.v. To qualify, children had to have FEV at $40 \%$ of predicted value. Although the researchers claimed that some physicians would add steroids and some would not, and that it was not known whether steroids would help, the committee, after further consultation with experts, judged that there may be a real risk in putting children with such severe asthma on placebo.

Case 4: new therapy not likely to be better than present therapy. Clinicians wanted to compare the routine therapy for primary osteomyelitis with IV cloxacillin to orally administered fusidic acid which is widely used in Europe. Children were to be randomized to receive one of the two. Analysis of available published reports did not reveal a well controlled randomized study in adults comparing the two modalities. Moreover, IV cloxacillin covers to date all staphylococcal osteomyelitis infections in children and, although the i.v. route may involve minor discomfort, the illness is serious and suboptimal therapy may result in longterm sequelae.

Case 5: emotionally invasive. Researchers who were not the initial surgeons to treat the child and, in fact, were never part of the team, wished to contact adolescent girls with congenital anorectal anomalies to inquire by the telephone about their sexual behavior. The committee believed that any study should be conducted by the personnel that treated the children. The nature of the questions were believed not to be appropriate to be asked through the telephone by an anonymous person. In addition to the potential emotional damage to the girls, the committee believed that such a procedure breaches medical confidentiality.

Case 6: emergency consent for randomization. This study aimed at comparing the efficacy of a new third-generation cephalosporin to that of the combination ampicillin-chloramphenicol (which at the time was the treatment of choice) for the treatment of bacterial meningitis in small children after the neonatal period. Parents would be asked to agree for randomization of their children at the emergency room soon after the diagnosis had been made. It was believed that this would put parents in an unacceptable level of anxiety, especially in view of the fact that the new medication was not expected at that time to have a better antibacterial potency.

Case 7: exemption from an information sheet. The researchers wished to assess through the telephone, the repetition of usage of the "baby walker" in infants who were already seen in the emergency room after injury caused by the use of the instrument. Although all parents would be asked for their consent for the telephone followup, it was believed that an information sheet specifying the objectives of the study would likely affect its results.

Case 8: consent by adolescents. The researchers wished to study the effects of OC hormones on the disposition and effects of drugs in adolescent girls receiving the "pill." These youngsters received the OC often without their parents' knowledge, and sometimes after termination of pregnancy. Some girls would be younger than $16 \mathrm{y}$ of age. The committee believed that by making the confidential choice of taking the $\mathrm{OC}$, these girls could be judged mature enough to consent for the study, which involved one dose of theophylline and blood sampling through an indwelling catheter. 